\title{
Updated recommendations regarding the management of older patients with breast cancer: a joint paper from the European Society of Breast Cancer Specialists (EUSOMA) and the International Society of Geriatric Oncology (SIOG)
}

Dr. Laura Biganzoli, MD

“Sandro Pitigliani” Department of Medical Oncology, Hospital of Prato, Prato, Italy

Dr. Nicolò Matteo Luca Battisti, MD

Breast Unit - Department of Medicine, The Royal Marsden NHS Foundation Trust, Downs Road, Sutton, London, United Kingdom

Breast Cancer Research Division, The Institute of Cancer Research, 15 Cotswold Road, Sutton, London, United Kingdom

Prof. Hans Wildiers, PhD

Department of General Medical Oncology, University Hospitals Leuven, Leuven, Belgium

Dr. Amelia McCartney, MBBS

“Sandro Pitigliani” Department of Medical Oncology, Hospital of Prato, Prato, Italy

Dr. Giuseppe Colloca, PhD

Unità Operativa Complessa di Radioterapia Oncologica, Dipartimento di Diagnostica per Immagini, Radioterapia Oncologica ed Ematologia, Fondazione Policlinico Universitario A. Gemelli IRCCS, Rome, Italy

Prof. Ian H. Kunkler, FRCR

Institute of Genetics and Molecular Medicine, Western General Hospital Campus, Crewe Road, Edinburgh, United Kingdom

Prof. Maria-João Cardoso, $\mathrm{PhD}$

Breast Unit, Champalimaud Clinical Center, Champalimaud Foundation and Nova Medical School, Lisbon, Portugal

Prof. Kwok-Leung Cheung, MD

School of Medicine, University of Nottingham, United Kingdom

Dr. Nienke Aafke de Glas, PhD

Leiden University Medical Center, Department of Medical Oncology, P.O. Box 9600, 2300 RC Leiden, The Netherlands 
Dr. Rubina M. Trimboli, MD

Unit of Radiology, Humanitas Clinical and Research Center, Rozzano, Italy

Prof. Beatriz Korc-Grodzicki, PhD

Memorial Sloan Kettering Cancer Center, New York, United States of America

Dr. Enrique Soto-Perez-de-Celis, MD

Department of Geriatrics, Instituto Nacional de Ciencias Medicas y Nutricion Salvador

Zubiran, Mexico City, Mexico

Dr. Antonio Ponti, MD

CPO Piemonte, AOU Città della salute e della scienza, Turin, Italy

Dr. Janice Tsang, MD

Hong Kong Breast Oncology Group, Li Ka Shing Faculty of Medicine, University of Hong Kong, Hong Kong

Dr. Lorenza Marotti, PhD

European Society of Breast Cancer Specialists (EUSOMA), Florence, Italy

Ms Karen Benn, BA

EUROPA DONNA - The European Breast Cancer Coalition, Milan, Italy

Dr. Matti S. Aapro, MD

Genolier Cancer Center, Clinique de Genolier, Switzerland

Dr. Etienne G.C. Brain, MD

Department of Medical Oncology, Institut Curie, Saint-Cloud and Paris, France

\section{Corresponding author:}

Dr. Laura Biganzoli, MD

"Sandro Pitigliani” Department of Medical Oncology

Hospital of Prato

Via Suor Niccolina Infermiera, 20, 59100 Prato PO, Italy

email: laura.biganzoli@uslcentro.toscana.it 


\section{Summary}

Breast cancer is increasingly prevalent in older adults in the context of ongoing demographic changes and is a significant part of routine oncology practice. Nonetheless, due to its highly heterogeneous nature, management of breast cancer in this population is challenging, with the validity of the available evidence very limited for older adults. Decision-making should not be driven by age alone but involve geriatric assessments plus careful consideration of life expectancy, competing risks of mortality, and patient preferences.

A multidisciplinary task force including members of the International Society of Geriatric Oncology (SIOG) and the European Society of Breast Cancer Specialists (EUSOMA) gathered to expand and update the previous 2012 evidence-based recommendations for the management of breast cancer in older individuals with the endorsement of the European Cancer Organisation. These were expanded to include chemotherapy toxicity prediction calculators, cultural and social considerations, surveillance imaging, genetic screening, genomic tools, neoadjuvant systemic treatment options, bone-modifying agents, targeted therapies and supportive care. Recommendations on geriatric assessment, ductal carcinoma in situ, screening, primary endocrine therapy, surgery, radiotherapy, adjuvant systemic therapy and secondary breast cancer were updated. 


\section{$\underline{\text { Introduction }}$}

Aging is the leading risk factor for cancer.(2) The prevalence of breast cancer (BC) in older adults is increasing and the higher cancer mortality in older adults compared with younger women establishes a major health disparity which may be explained by more advanced presentation, delayed diagnosis, organ function decline and multimorbidities.(3) Nonetheless, functional age (and not chronological age) and the potential underlying frailty should drive decision-making. Older patients are underrepresented in clinical trials which do not always enrol individuals more frequently seen in routine practice. Therefore, the risks and benefits of anticancer therapy should be carefully weighed.(4)

A multidisciplinary task force including specialists in medical oncology, radiation oncology, surgery, geriatrics, radiology and epidemiology and patient advocates affiliated with the International Society of Geriatric Oncology (SIOG) was created in 2007 to prepare recommendations for the management of BC in older individuals.(5) These were subsequently updated in 2012 in collaboration with the European Society of Breast Cancer Specialists (EUSOMA).(6) Here we present an update of the task force recommendations based on the new evidence which has become available since 2012 (Table 1). These recommendations are a consensus by an expert task force on available evidence and expert opinion. 


\section{Search strategy, selection criteria and grading of the evidence}

Each task force expert performed a scoping literature review on Pubmed/Medline on individual topics pertaining to breast oncology (MeSH: "older" or "elderly" and "breast cancer" and "surgery", "radiotherapy" or "systemic therapy") and any updates available since the previous recommendations were published in April 2012. The list of topics included epidemiology, geriatric assessment, cultural and social considerations, genetic screening, ductal carcinoma in situ, screening, surveillance imaging, primary endocrine therapy, surgery, radiotherapy, adjuvant and neoadjuvant systemic therapy, genomic tools, treatment of secondary breast cancer, chemotherapy toxicity prediction, bone-modifying agents, targeted therapies and supportive care. The experts presented the results of each individual scoping review to the task force during various meetings held between February 2019 and August 2020. During these meetings, the need to update the previous recommendations was discussed and consensus reached by unanimity; the level of evidence was graded according to the four-classes classification proposed by the US Agency for Healthcare Research and Quality (AHRQ) and recently adopted by EUSOMA.(1)

\section{General and worldwide concepts on ageing}

Frailty involves decreased physiological and functional reserve leading to vulnerability to stressors and adverse outcomes. Strayifying patients as fit, vulnerable and frail may identify those at risk of complications.(7) Collaboration between cancer specialists and geriatricians and geriatric assessment (GA) are recommended. Frail individuals require tailored approaches based on a GA and focusing on supportive care. Fit individuals may tolerate standard treatment similarly to younger patients. Vulnerable individuals may require treatment adjustments and geriatric interventions. Competing mortality risks may justify less aggressive approaches. The National Comprehensive Cancer Network (NCCN) and American Society of Clinical Oncology (ASCO) guidelines recommend evaluating life expectancy and calculators such as ePrognosis may aid in assessing whether cancer is likely to shorten it. $(8,9)$ Since competing mortality risks are more prevalent in older adults even without multimorbidities, treatment decisions should consider not only the risk of $\mathrm{BC}$ recurrence, but also the risk of dying of other causes, which is strongly influenced by frailty. 
GA is a multidimensional evaluation aiming to determine physiologic age and guide diagnostic and therapeutic interventions targeting reversible deficits and devisising treatment strategies to eliminate or mitigate them.(10) Increasing evidence supports the role of GA in the care of older patients with BC. The implementation of GA may improve tolerance, health-related quality of life (QoL) and satisfaction.(11-15) ASCO recommends GA for patients aged 65 years and older considered for chemotherapy.(8) GA can be time-consuming and may not be necessary for all older patients. Several screening tools (some self-reported) can identify patients requiring GA, and should be considered as the gateway to any cancer treatment decision-making in patients aged 70 and older. $(16,17)$

The Cancer and Aging Research Group (CARG) and the Chemotherapy Risk Assessment Scale for High-age patients (CRASH) scores estimate the risk of grade 3-5 chemotherapy toxicity in older patients (Table 2) and were validated in cohorts including $20 \%$ of BC patients. $(18,19) \mathrm{A}$ BC-specific risk score (CARG-Breast) has been developed and validated but is not yet available.(20) Chemotherapy toxicity calculators should be used as an adjunct in the decisionmaking process.(21) Multimorbidity and toxicity may influence treatment efficacy (especially endocrine therapy) as nonadherence increases with age.(22)

Cultural and social aspects, including religious myths and taboos, and patient values must be considered during diagnosis and treatment, especially in the context of the current migration flows. Older adults from immigrant populations may have more disabilities, worse self-rated health and poorer outcomes. Literacy and education are also heterogeneous and some assessment tools may not be universally applicable.

\section{Mammography screening and surveillance}

\section{Screening}

Most screening programs extend until 69-70 years and a minority until 74-75 years. The European Commission Initiative on Breast Cancer and the US Preventive Services Task Force recommend screening mammography for women aged 70-74 years despite the risk of overdiagnosis. $(23,24)$ A meta-analysis found a relative risk reduction for BC mortality of 0.80 for women aged 70-74 years, (25) although there is controversy also in younger patients. Screening every 2-3 years is deemed to provide the best balance between benefits and harms. The American Cancer Society recommends mammography in older women,(26) particularly in the 
context of a life expectancy $\geq 10$ years. However, screening is unlikely to be beneficial after age 75(24) and decisions should consider overall health and life expectancy.

\section{Surveillance}

No evidence supports the benefit of mammographic surveillance on disease-specific mortality for older BC survivors in the context of multimorbidities and competing mortality risks. The risk for ipsilateral recurrence and contralateral BCs over the age of 75 years is not defined and is influenced by tumour biology and adjuvant therapy.(27) International guidelines recommend indefinite annual mammography regardless of age. $(9,28)$ Annual or biennial mammography is recommended for women aged 70-80 years although multimorbidities, life expectancy and frailty should be considered.(27) It should be avoided in patients over 80 years with multimorbidities or life expectancy $\leq 5$ years.(29)

\section{Genetic screening and its implications}

The prevalence of pathogenic variants associated with a germline $\mathrm{BC}$ predisposition is almost 3 times less over age 65 (5.6\% vs 14.2\%).(30) BRCA2 and CHEK2 have been found to be relatively prevalent in women aged over 65 with BC.(30) Nonetheless, they are less likely to undergo genetic testing, as guidelines often focus on younger populations. For older patients, genetic testing based on simple, cancer-based criteria may potentially deliver consistent, costeffective and patient-centred outcomes. Selection of candidates appropriate for screening should be considered in line with current local and/or national guidelines.

In the curative setting, germline pathologic variant carriers may benefit from high-risk surveillance or risk-reducing interventions in the context of an adequate life expectancy.(9) Also, carriers should be offered cascade testing and evaluation of their relatives. For advanced disease, poly ADP-ribose polymerase (PARP) inhibition is a potential alternative to chemotherapy for older BRCA carriers, especially regarding QoL.(31)

\section{Neoadjuvant systemic therapy}

Fit older patients should be considered for neoadjuvant strategies similarly to their younger counterparts based on the clinical subtypes of the primary tumour.(32) Due to the higher risk of adverse outcomes,(33-35) vulnerable patients may be better served by upfront surgery, 
particularly if $\mathrm{BC}$ is already operable. The likelihood of breast conservation should also be considered based on disease characteristics, expected response and patient preference. In fit older persons with high-grade triple negative BC (TNBC), optimal chemotherapy is still debated. Similarly to the adjuvant setting, sequential regimens with anthracyclines and taxanes may be considered although evidence is very limited and shorter regimens remain reasonable. Adding platinum compounds remains debated and may be challenging for most older adults.

Pathological response after neoadjuvant chemotherapy may guide adjuvant treatment decisions for TNBC and human epidermal growth factor receptor 2 (HER2)-positive BC.(36, 37) The CREATE-X and KATHERINE trials enrolled few older individuals but did not show any new safety concerns. Therefore, fit older patients should be considered for such approaches in case of residual disease.

Neoadjuvant endocrine therapy (ET) is associated with lower toxicity, reasonable response rates, and similar breast-conservation rates as neoadjuvant chemotherapy, but survival data are not available. This approach may be useful in older patients not deemed suitable for upfront surgery pending preoperative assessments. Aromatase inhibitors (AI) are recommended over tamoxifen due to improved clinical and radiological response and breast conservation rates.(38) A course of 4-6 months should be considered.

\section{Surgery}

While surgery remains the standard treatment in most older patients with early disease, there is a risk of over-treatment with competing mortality risks warranting the use of GA and survival estimates before proceeding with it.(39) However, BC surgery is generally safe, whereas endocrine therapy may cause side effects potentially impacting QoL.(22)

\section{Surgery or not}

Two systematic reviews demonstrate a local control and survival benefit with surgery over primary endocrine therapy (PET) in patients with a life expectancy $\geq 5$ years. $(40,41)$ However, in a large cohort study, no BC-specific survival differences were seen between surgery and PET in strong hormone receptor (HR)-positive disease.(42) When PET involves aromatase inhibitors (AIs), the median time to progression is approximately five years.(42) The benefit of PET versus upfront surgery is expected to be more pronounced with a life expectancy of less than 5 years. 


\section{Ductal carcinoma in-situ (DCIS)}

Opportunistic screening exposes older patients to potential over-diagnosis and over-treatment of DCIS. Ongoing non-intervention trials will define the role of 'watch and wait' approaches. Meanwhile, fit patients with high-grade DCIS and no multimorbidities should undergo surgery. In low- and intermediate-grade DCIS, surgery and/or postoperative radiotherapy may be spared based on life expectancy and competing risks.(43)

\section{Surgery to the axilla}

Less invasive approaches to the axilla in case of $\mathrm{cNO}$ disease are particularly relevant for older adults. Axillary clearance does not produce any survival benefit, and in older patients regional recurrences without axillary surgery remains rare.(44) Therefore, in older adults, sentinel node biopsy (SNB) should be 'standard' for clinically/radiologically node-negative axillae. In most cases further axillary surgery can be avoided if only 1-2 sentinel nodes are involved(45) or replaced by radiotherapy.(46) As even SNB is associated with side effects and likely does not improve prognosis by itself, omission of axillary staging by SNB may be appropriate for frail individuals with low-volume, luminal A-like tumours.

\section{Oncoplastic and reconstructive surgery}

Oncoplastic and reconstructive surgery are offered less frequently to older patients.(47) Some older patients may decline such approaches more frequently compared with their younger counterparts, but their personal preferences should be balanced with risks. Oncoplastic and reconstructive procedures may be reasonable alternatives to simple mastectomy or breast conservation.(47) The pros and cons of complex versus simpler procedures should be carefully assessed and discussed with patients.

\section{Radiotherapy}

Radiotherapy after breast conserving surgery

Postoperative whole breast radiotherapy (WBRT) halves the risk of first recurrence and remains standard-of-care for most older patients following breast conserving surgery (BCS).(48) However, the absolute benefit in older patients with low-grade, HR-positive disease is modest. Omission of radiation therapy (RT) remains controversial. The CALGB 9343 trial 
showed a loco-regional recurrence rate without RT of $10 \%$, versus $2 \%$ with RT after 12 years of follow-up in women aged over 70, with no detrimental impact on OS, and these relapses could be corrected successfully by second and deferred surgery.(49) The PRIME II trial showed a lower risk of ipsilateral breast tumour recurrence (IBTR) at 5 years for those receiving WBRT.(50) Both studies suggest omitting radiotherapy in low-risk patients may be reasonable and the results of the PRIMETIME study are awaited. Recommendations regarding radiotherapy omission in low-risk patients from the 2017 NCCN and National Institute for Care and Clinical Excellence guidelines are presented in Table 3.

\section{Tumour bed boost}

In the EORTC boost/no boost trial,(51) the relative risk reduction was not statistically significant for patients aged over 60 years. Therefore, a boost is advised in this age group only in case of a higher risk of recurrence.

\section{Partial breast irradiation}

No trials of partial breast irradiation (PBI) focused specifically on older patients. The GECESTRO trial of multicatheter brachytherapy versus WBRT suggested that PBI is not inferior to WBRT.(52) The UK IMPORT-LOW trial showed that partial breast and reduced dose EBRT is non-inferior to standard WBRT, with equivalent or fewer side effects.(53) The UK consensus recommends PBI to women aged $\geq 50$ years or with grade $1-2$, pN0, HR-positive, HER2negative, tumours $\leq 30 \mathrm{~mm}$ and with radial margins $\geq 1 \mathrm{~mm}$.(54)

\section{Regional nodal irradiation}

Three randomised controlled trials show the benefit of regional nodal irradiation (RNI) in highrisk early BC, $(46,55,56)$ however none specifically focused on older patients. RNI is indicated in patients with 4 or more positive nodes, but it is unclear which group of patients with 1-3 positive nodes benefit from it.(57)

\section{Postmastectomy radiotherapy}

Evidence supporting the role of postmastectomy radiotherapy (PMRT) in older women is lacking and recommendations are extrapolated from analyses conducted in younger patients. PMRT is standard of care in patients with $\geq 4$ positive nodes, whilst the role of PMRT in patients with 1-3 positive nodes remains controversial. An EBCTCG meta-analysis showed PMRT reduced 20-year BC-mortality by $7.9 \%$ for patients with 1-3 positive lymph nodes and 
by $9.3 \%$ for patients with $\geq 4$ positive lymph nodes.(48) Therefore, some argue that PMRT should be standard for all node-positive patients, while others question its role in the context of current treatment approaches. Specific guidelines are available. $(9,58,59)$ The BIG 2-04 MRC SUPREMO trial evaluating PMRT in patients with 1-3 positive nodes or pN0 with LVI/grade 3 with no upper age limit remains in follow-up phase.(60) While NICE and NCCN guidelines suggest that decision-making should be driven by nodal disease burden, $(58,61)$ the ASCO-ASTRO-SSO recommendations highlight the relevance of age, life expectancy, multimorbidities, tumour burden and biology.(59)

\section{Dose fractionation schedules after breast conserving surgery or mastectomy}

Hypofractionated schedules are recommended for older as in younger patients as per the FAST FORWARD study results.(62)

\section{Adjuvant systemic therapy}

\section{Adjuvant chemotherapy in older adults with HER2-negative disease}

BC subtype and stage are key in informing adjuvant chemotherapy decisions. Prospective trials(63) and large retrospective cohorts $(64,65)$ confirm the potential large benefit of adjuvant chemotherapy on BC-specific survival or overall survival mostly in ER-negative disease, irrespective of nodal status. A recent retrospective study showed OS benefit in patients aged $\geq 70$ years with node-positive, ER-positive, HER2-negative BC, also with comorbidities, (66) despite selection bias remains a significant limitation. For luminal disease, genomic tools may identify those who might benefit from chemotherapy. However, most gene expression assay validation studies excluded older patients and do not address competing risks. OncotypeDx ${ }^{\circledR}$ remains the most frequently studied tool in this age group. Its prognostic accuracy is not influenced by age, but disappointingly a high RS does not predict adjuvant chemotherapy benefit in older patients.(67) Therefore, integrating general health status with gene prognostic models is essential. Nonetheless, although results should be interpreted cautiously, this should not disqualify older patients from such tests. The ASTER 70s study will clarify the role of tumour genomic data in older BC patients.

Online prediction tools are affordable but have substantial limitations in older patients.(68) NHS PREDICT is accurate in older patients only when predicting outcomes at 5 years (but not 
at 10 years) and is not reliable in the presence of multimorbidities and over 80 years.(69) Additionally, it estimates survival but not the risk of recurrence. The Age Gap Decision Tool is promising in comparing local treatment with or without chemotherapy but requires prospective validation (https://agegap.shef.ac.uk/).

\section{Chemotherapy regimen choice}

Although no evidence supports differential use of adjuvant chemotherapy, older adults may experience more frequent adverse events including death.(70) Benefits of adjuvant combination chemotherapy are maintained at least up until age 70, although biased by chemotherapy duration(71) and limited to HR-negative and/or node-positive disease.(65)

Modified regimens should not be utilised in older patients (Table 4). The CALGB 49907 trial showed significantly worse survival with capecitabine versus standard regimens (four cycles of doxorubicin/cyclophosphamide [AC] or six cycles of cyclophosphamide/methotrexate/fluorouracil [CMF]) in older women, with a high interaction of ER status and competing risks diluting overall survival benefits with longer follow-up.(63) The ELDA trial demonstrated worse QoL with docetaxel versus CMF and no survival benefit.(72)

Older adults were excluded or highly selected in trials of sequential anthracycline and taxanebased regimens, which should be considered only in fit patients with large, node-positive, triple-negative tumours. Dose-dense regimens should not be utilised based on the increased toxicity risk and the lack of efficacy data in older persons. In many older patients, four cycles of docetaxel/cyclophosphamide (TC) may be appropriate, which is superior to AC and more tolerable.(73) Weekly paclitaxel may be considered for high-risk patients unfit for polychemotherapy. Table 4 illustrates common chemotherapy regimens that may be considered.

\section{Safety of adjuvant chemotherapy in older adults}

Older patients have higher risk of chemotherapy toxicity and mortality.(74) Risks include haematological toxicity, anthracycline-associated cardiotoxicity (occurring in up to 38\%), taxane-related neurotoxicity, falls, decreased QoL, and hospitalisations. However, functional 
decline and impaired QoL may be temporary.(75) Long-term consequences include musculoskeletal events, acute myeloid leukaemia/myelodysplastic syndrome, cognitive decline, and impaired function. Chemotherapy duration (double for sequential versus singleagent regimens) should be limited, with a 3-month threshold for increased serious side effects.(20)

\section{Anti-HER2 treatment in adjuvant setting}

Although adjuvant trastuzumab is beneficial regardless of age,(76, 77) anti-HER2 (neo)adjuvant strategies remain poorly investigated in patients $\geq 65$ years. Pertuzumab may be considered for high-risk individuals,(37) but diarrhoea may be debilitating in older adults, as with adjuvant neratinib (Table 4).

SIOG recommends adjuvant chemotherapy along with one year of trastuzumab as a standard approach in older patients with normal cardiac function and early-stage HER2-positive BC larger than $0.5 \mathrm{~cm}$, and consideration of pertuzumab only in selected high-risk and fit patients (Table 4).(78) The preferred chemotherapy backbone includes four cycles of TC or weekly paclitaxel. Although evidence is scarce, omission of chemotherapy and utilisation of singleagent trastuzumab (plus endocrine therapy if indicated),(79) may be appropriate in vulnerable and frail patients.(78) A shorter course of adjuvant anti-HER2 therapy may also be considered for older patients with small, node-negative disease or cardiac problems.

\section{Safety of anti-HER2 therapy in older persons}

Age correlates with higher cardiac toxicity rates on trastuzumab,(80) with $15-40 \%$ of patients requiring early discontinuation especially $\geq 80$ years of age and with multimorbidities,(81) likely predominantly due to chemotherapy-related adverse events. However, up to one third of cardiac events occur within two years of treatment completion, which may be more specifically related to trastuzumab.

\section{Role of adjuvant endocrine treatment}


All postmenopausal women suitable for ET should be offered endocrine therapy regardless of age. However, ET may be omitted in the absence of any documented impact on mortality in patients with very low-risk disease and/or short life expectancy.(82)

\section{Choice of agent}

Selection of agents should take into account multimorbidities and recurrence risk. AIs result in slightly better reduction in recurrence and BC-specific mortality compared to tamoxifen, and are preferable upfront especially in high-risk patients.(83) Following a few years of AIs, switching to tamoxifen is similarly effective to their continuation. Musculoskeletal side effects may impair adherence to AIs Long-term problems may include osteoporosis, cardiovascular risk, diabetes, hypercholesterolemia and cognitive impairment. Conversely, AIs are associated with a lower risk of venous thrombosis, endometrial cancer and fatty liver disease compared to tamoxifen. Good compliance should drive treatment decisions.

\section{Duration of therapy}

Letrozole improves survival outcomes versus placebo among patients who receive an initial five-year course of tamoxifen. After five intial years of AIs, data are less clear: a recurrencefree survival (RFS) benefit is not confirmed in all studies although bone-related adverse events are more frequent. The more modest impact on RFS and the impact on bone health is confirmed by large meta-analyses. Therefore, the current standard of care should include five years of ET, and extended therapy may be offered to fit, healthy older women with high-risk disease who tolerated the first five years.(84) In frail patients, recommendations should be guided by the individual circumstances.

\section{Role of adjuvant bone modifying agents}

Adjuvant systemic therapies for $\mathrm{BC}$ are associated with an increased risk of bone loss. Therefore, a baseline assessment of bone mineral density (BMD) in older patients suitable for adjuvant endocrine therapy is mandatory, followed by calcium and vitamin D supplementation and use of bisphosphonates to preserve bone mass while on AIs. Also, adjuvant bisphosphonates also improve survival outcomes in patients with early-stage disease.(85) An 
EBCTCG meta-analysis documented a 2-3\% benefit in BC-mortality limited to postmenopausal women receiving bisphosphonates.(86)

Zoledronate or clodronate should be offered regardless of age to postmenopausal women with moderate- to high-risk BC according to international consensus. Evidence is insufficient for alendronate and risedronate. Bisphosponate use should take into account the minor improvement in long-term survival and their potential side effects, including electrolyte disturbances (mostly hypocalcemia), atypical fractures and osteonecrosis of the jaw, $(87,88)$ multimorbidities, renal function, fitness and patient preferences. The role of denosumab is controversial and should not be considered in the adjuvant setting for older patients to reduce mortality. The ABCSG-18 study showed improved DFS and bone fracture rate in patients on adjuvant denosumab(89) but the subsequent D-CARE study failed to detect any benefit in bone metastasis-free survival or DFS.(90) Additionally, a rebound effect with more vertebral fractures occurring upon its discontinuation has been demonstrated.

\section{Systemic treatment for metastatic disease}

Different treatment schedules, dose reductions or stepwise dose-escalation before reaching standard recommended dose might be required in older patients(91) and reduce the risk of adverse outcomes.

\section{Chemotherapy}

Chemotherapy should be considered in suitable older patients with HR-negative disease, HRpositive disease resistant to ET or with rapidly progressive disease and/or extensive visceral involvement and based on GA and patient preferences. The increased toxicity risk in this age group mandates particular attention to minimising side effects.(8) Single-agent regimens are preferred over polychemotherapy(6) and chemotherapy toxicity prediction tools may also be useful. Preference should be given to agents studied in older populations. Nab-paclitaxel is associated with very few allergic reactions, does not require steroids and is safe and effective in patients over 65.(92) Following anthracyclines or taxanes, eribulin is also appropriate, with similar efficacy and toxicity regardless of age and no impact on GA parameters nor QoL.(93) 


\section{HER2-positive metastatic breast cancer}

Older patients with HER2-positive metastatic BC and adquate cardiac function should receive HER2-directed therapy based on fitness.(78) Although docetaxel or paclitaxel in combination with trastuzumab and pertuzumab are recommended in fit patients, taxanes may cause severe toxicities. In older patients not suitable for taxanes, capecitabine or vinorelbine may be considered. The EORTC 75111-10114 study(94) enrolling older patients evaluated trastuzumab and pertuzumab with or without metronomic oral cyclophosphamide. Vinorelbine along with dual anti-HER2 blockade may also be considered.

ET with trastuzumab plus pertuzumab or lapatinib is a reasonable alternative for patients with ER-positive disease, despite diarrhoea may be an issue requiring close monitoring. T-DM1 is recommended in later therapy lines in fit older patients, but further research in frail patients is warranted.

\section{Targeted agents in luminal tumours}

Efficacy of cyclin-dependent kinases 4/6 (CDK4/6) inhibition is age-independent in the subgroup and pooled analyses of the landmark studies of palbociclib, ribociclib and abemaciclib,(95-98) with no age-related changes in pharmacokinetics. Nevertheless, patients $\geq 75$ years experience higher rates of toxicity and dose modifications.(98) While ET alone is still reasonable in selected cases, CDK4/6 inhibitors are a suitable treatment in older patients.(99)

Everolimus should be used with caution in older patients in view of its safety profile. A subgroup analysis of the BOLERO-2 study revealed a higher rate of discontinuations in patients $\geq 70$ years and more on-treatment deaths.(100) $26 \%$ of patients enrolled in the expanded-access BALLET trial were aged $\geq 70$, which similarly reported more frequent AErelated dose discontinuations, reductions and interruptions.

\section{Supportive care}

Supportive care is important as cancer and its treatment can seriously harm and lead to various degrees of decompensation of older patients. For detailed guidance, the reader can also consult 
the ESMO, MASCC and SIOG websites (https://www.esmo.org/; https://www.mascc.org/; https://www.siog.org/).

\section{Digestive symptoms}

Nausea and vomiting can be treatment-related or have alternative aetiologies. In older individuals, diagnosis may be challenging as clinical signs may be absent or atypical. Guidelines for prevention of chemotherapy and radiation therapy-induced nausea and vomiting should be followed. General management guidelines for diarrhoea, constipation and stomatitis are available.

\section{Malnutrition}

More than $30 \%$ of older patients experience severe malnutrition in the hospital and nursing home settings. Malnutrition can lead to osteopenia/osteoporosis, sarcopenia, immunological deficiencies and iron, vitamin B12 or folate-related anaemia, and predicts outcomes at three years. This may be improved by timely intervention.

\section{Depression}

Depression in older cancer patients is often under-recognised and untreated but can be successfully managed with psychological support, and antidepressants when indicated. Drug interactions should be considered, such as those between selective serotonin-reuptake inhibitors and tamoxifen.

\section{Pain control}

Pain can be related to or complicated by multimorbidities such as arthritis or osteoporotic fractures. Older patients are generally more susceptible to changes in drug doses, side effects, and drug interactions. Particular attention should be paid to potential side effects of nonsteroidal anti-inflammatory drugs (renal function, gastric ulcers). Guidelines are available, with the above caveats.

\section{Febrile neutropenia prevention and treatment}

Guidelines on the primary prophylactic use of white blood cell growth factors acknowledge the increased risk of myelosuppression in individuals aged $>65$. In the general population, the febrile neutropenia risk threshold of $\geq 20 \%$ is for consideration of primary prophylaxis, but for older persons, a lower threshold may be used, e.g. $>10 \%$, which is reached in older persons when using standard myelosuppressive regimens as anthracyclines or TC. 


\section{Conclusions}

The management of $\mathrm{BC}$ in older adults should involve routine use of GA tools and close interaction with members of the multidisciplinary team due to the intrinsic heterogeneity of this population. In the context of the limited applicability of the evidence generated in younger and/or more fit individuals, patient preferences, life expectancy, predicted survival benefits and impact on toxicity and QoL should be carefully considered in decision-making. 


\section{Conflict of interest statement}

Dr. Biganzoli reports personal fees from AstraZeneca, grants, personal fees and non-financial support from Celgene, personal fees from Daiichi-Sankyo, personal fees from Eisai, grants and personal fees from Genomic Health, personal fees and non-financial support from Ipsen, personal fees from Lilly, grants and personal fees from Novartis, personal fees and nonfinancial support from Pfizer, personal fees from Pierre Fabre, personal fees and non-financial support from Roche, outside the submitted work. Dr. Battisti reports grants and personal fees from Pfizer, grants from Genomic Health, outside the submitted work. Dr. Wildiers reports that his institution received consulting fees and honoraria from AstraZeneca, Biocartis, Lilly, Novartis, Pfizer, PUMA Biotechnology, Roche, Sirtex, Daiiji. His institution received unrestricted research grants from Roche and Novartis. He received travel support from Roche and Pfizer. Dr. McCartney has nothing to disclose. Dr. Colloca has nothing to disclose. Dr. Kunkler has nothing to disclose. Dr. Cardoso has nothing to disclose. Prof. Cheung has nothing to disclose. Dr. de Glas has nothing to disclose. Dr. Trimboli has nothing to disclose. Dr. KorcGrodzicki has nothing to disclose. Dr. Soto-Perez-de-Celis has nothing to disclose. Dr. Ponti has nothing to disclose. Dr. Tsang has nothing to disclose. Dr. Marotti has nothing to disclose. Dr. Benn has nothing to disclose. Dr. Aapro reports personal fees and non-financial support from Multinational Association for Supportive Care in Cancer, personal fees and non-financial support from European Society of Medical Oncology, personal fees and non-financial support from European CanCer Organisation, grants and personal fees from Helsinn, personal fees from Tesaro, grants and personal fees from Sandoz, personal fees from Merck USA, personal fees from Vifor, personal fees from Pfizer, personal fees from Taiho, personal fees from Kyowa Kirin, outside the submitted workDr. Brain reports personal fees and other from Pfizer, personal fees and other from Roche, other from Pierre Fabre, grants, personal fees and other from BMS, personal fees from Samsung, other from Novartis, other from AstraZeneca, personal fees from TLC PharmaChem, personal fees from Clinigen, personal fees from Mylan, personal fees from G1 Therapeutics, outside the submitted work.

No authors are employed by NIH.

\section{Contributions}

All authors contributed equally to literature search, figures, data interpretation and writing. 


\section{Disclosure}

This paper has not been submitted to another journal and has not been published in whole or in part elsewhere previously. 


\section{References}

1. Biganzoli L, Marotti L, Hart CD, Cataliotti L, Cutuli B, Kühn T, et al. Quality indicators in breast cancer care: An update from the EUSOMA working group. Eur J Cancer. 2017;86:59-81.

2. Siegel RL, Miller KD, Jemal A. Cancer statistics, 2019. CA Cancer J Clin. 2019;69(1):7-34.

3. Bagegni NA, Peterson LL. Age-related disparities in older women with breast cancer. Adv Cancer Res. 2020;146:23-56.

4. Hurria A, Levit LA, Dale W, Mohile SG, Muss HB, Fehrenbacher L, et al. Improving the Evidence Base for Treating Older Adults With Cancer: American Society of Clinical Oncology Statement. J Clin Oncol. 2015;33(32):3826-33.

5. Wildiers H, Kunkler I, Biganzoli L, Fracheboud J, Vlastos G, Bernard-Marty C, et al. Management of breast cancer in elderly individuals: recommendations of the International Society of Geriatric Oncology. Lancet Oncol. 2007;8(12):1101-15.

6. Biganzoli L, Wildiers H, Oakman C, Marotti L, Loibl S, Kunkler I, et al. Management of elderly patients with breast cancer: updated recommendations of the International Society of Geriatric Oncology (SIOG) and European Society of Breast Cancer Specialists (EUSOMA). Lancet Oncol. 2012;13(4):e148-60.

7. Guerard EJ, Deal AM, Chang Y, Williams GR, Nyrop KA, Pergolotti M, et al. Frailty Index Developed From a Cancer-Specific Geriatric Assessment and the Association With Mortality Among Older Adults With Cancer. J Natl Compr Canc Netw. 2017;15(7):894-902.

8. Mohile SG, Dale W, Somerfield MR, Schonberg MA, Boyd CM, Burhenn PS, et al. Practical Assessment and Management of Vulnerabilities in Older Patients Receiving Chemotherapy: ASCO Guideline for Geriatric Oncology. J Clin Oncol. 2018;36(22):2326-47.

9. NCCN. NCCN clinical practice guidelines in oncology version 3.2019 genetic/familial

high-risk assessment: breast and ovarian.; 2019.

10. Wildiers H, Heeren P, Puts M, Topinkova E, Janssen-Heijnen ML, Extermann M, et al. International Society of Geriatric Oncology consensus on geriatric assessment in older patients with cancer. J Clin Oncol. 2014;32(24):2595-603.

11. Mohile SG, Epstein RM, Hurria A, Heckler CE, Canin B, Culakova E, et al. Communication With Older Patients With Cancer Using Geriatric Assessment: A Cluster-Randomized Clinical Trial From the National Cancer Institute Community Oncology Research Program. JAMA Oncol. 2019;6(2):1-9.

12. Li D, Sun C-L, Kim H, Chung V, Koczywas M, Fakih M, et al. Geriatric assessment-driven intervention (GAIN) on chemotherapy toxicity in older adults with cancer: A randomized controlled trial. Journal of Clinical Oncology. 2020;38(15_suppl):12010-.

13. Mohile SG, Mohamed MR, Culakova E, Xu H, Loh KP, Magnuson A, et al. A geriatric assessment (GA) intervention to reduce treatment toxicity in older patients with advanced cancer: A University of Rochester Cancer Center NCI community oncology research program cluster randomized clinical trial (CRCT). Journal of Clinical Oncology. 2020;38(15_suppl):12009-.

14. Qian CL, Knight HP, Ferrone CR, Kunitake H, Castillo CF-d, Lanuti M, et al. Randomized trial of a perioperative geriatric intervention for older adults with cancer. Journal of Clinical Oncology. 2020;38(15_suppl):12012-.

15. Soo W-K, King M, Pope A, Parente P, Darzins P, Davis ID. Integrated geriatric assessment and treatment (INTEGERATE) in older people with cancer planned for systemic anticancer therapy. Journal of Clinical Oncology. 2020;38(15_suppl):12011-.

16. Decoster L, Van Puyvelde K, Mohile S, Wedding U, Basso U, Colloca G, et al. Screening tools for multidimensional health problems warranting a geriatric assessment in older cancer patients: an update on SIOG recommendations $\dagger$. Ann Oncol. 2015;26(2):288-300.

17. Hamaker ME, Jonker JM, de Rooij SE, Vos AG, Smorenburg CH, van Munster BC. Frailty screening methods for predicting outcome of a comprehensive geriatric assessment in elderly patients with cancer: a systematic review. Lancet Oncol. 2012;13(10):e437-44.

18. Extermann M, Boler I, Reich RR, Lyman GH, Brown RH, DeFelice J, et al. Predicting the risk of chemotherapy toxicity in older patients: the Chemotherapy Risk Assessment Scale for High-Age Patients (CRASH) score. Cancer. 2012;118(13):3377-86. 
19. Hurria A, Togawa K, Mohile SG, Owusu C, Klepin HD, Gross CP, et al. Predicting chemotherapy toxicity in older adults with cancer: a prospective multicenter study. J Clin Oncol. 2011;29(25):3457-65.

20. Hurria A, Magnuson A, Gross CP, Tew WP, Klepin HD, Wildes TM, editors. Development and validation of a chemotherapy toxicity (Chemo Tox) risk score for older patients (pts) with breast cancer (BC) receiving adjuvant/neoadjuvant treatment (Adjuvant Tx): A R01 and BCRF funded prospective multicenter student [abstract]. 2018 San Antonio Breast Cancer Symposium; 20182018 Dec 4-8; San Antonio, TX.

21. Soto-Perez-de-Celis E, Li D, Yuan Y, Lau YM, Hurria A. Functional versus chronological age: geriatric assessments to guide decision making in older patients with cancer. Lancet Oncol. 2018;19(6):e305-e16.

22. van de Water W, Bastiaannet E, Hille ET, Meershoek-Klein Kranenbarg EM, Putter H, Seynaeve CM, et al. Age-specific nonpersistence of endocrine therapy in postmenopausal patients diagnosed with hormone receptor-positive breast cancer: a TEAM study analysis. Oncologist. 2012;17(1):55-63.

23. de Glas NA, de Craen AJ, Bastiaannet E, Op 't Land EG, Kiderlen M, van de Water W, et al. Effect of implementation of the mass breast cancer screening programme in older women in the Netherlands: population based study. Bmj. 2014;349:g5410.

24. García-Albéniz X, Hernán MA, Logan RW, Price M, Armstrong K, Hsu J. Continuation of Annual Screening Mammography and Breast Cancer Mortality in Women Older Than 70 Years. Ann Intern Med. 2020;172(6):381-9.

25. Nelson HD, Fu R, Cantor A, Pappas M, Daeges M, Humphrey L. Effectiveness of Breast Cancer Screening: Systematic Review and Meta-analysis to Update the 2009 U.S. Preventive Services Task Force Recommendation. Ann Intern Med. 2016;164(4):244-55.

26. Bredemeyer M. ACS Releases Guideline on Breast Cancer Screening. Am Fam Physician. 2016;93(8):711-2.

27. Freedman RA, Keating NL, Partridge AH, Muss HB, Hurria A, Winer EP. Surveillance Mammography in Older Patients With Breast Cancer-Can We Ever Stop?: A Review. JAMA Oncol. 2017;3(3):402-9.

28. Runowicz CD, Leach CR, Henry NL, Henry KS, Mackey HT, Cowens-Alvarado RL, et al. American Cancer Society/American Society of Clinical Oncology Breast Cancer Survivorship Care Guideline. CA Cancer J Clin. 2016;66(1):43-73.

29. Brownlee S, Chalkidou K, Doust J, Elshaug AG, Glasziou P, Heath I, et al. Evidence for overuse of medical services around the world. Lancet. 2017;390(10090):156-68.

30. Chavarri-Guerra Y, Hendricks CB, Brown S, Marcum C, Hander M, Segota ZE, et al. The Burden of Breast Cancer Predisposition Variants Across The Age Spectrum Among 10000 Patients. J Am Geriatr Soc. 2019;67(5):884-8.

31. Liposits G, Loh KP, Soto-Perez-de-Celis E, Dumas L, Battisti NML, Kadambi S, et al. PARP inhibitors in older patients with ovarian and breast cancer: Young International Society of Geriatric Oncology review paper. J Geriatr Oncol. 2019;10(2):337-45.

32. Mieog JS, van der Hage JA, van de Velde CJ. Preoperative chemotherapy for women with operable breast cancer. Cochrane Database Syst Rev. 2007(2):Cd005002.

33. Barcenas CH, Niu J, Zhang N, Zhang Y, Buchholz TA, Elting LS, et al. Risk of hospitalization according to chemotherapy regimen in early-stage breast cancer. J Clin Oncol. 2014;32(19):2010-7.

34. Pinder PC, Duan Z, Goodwin JS, Hortobagyi GN, Giordano SH. Congestive Heart Failure in Older Women Treated With Adjuvant Anthracycline Chemotherapy for Breast Cancer. Journal of Clinical Oncology. 2007;25(25):3808-15.

35. Rosenstock AS, Lei X, Tripathy D, Hortobagyi GN, Giordano SH, Chavez-MacGregor M. Short-term mortality in older patients treated with adjuvant chemotherapy for early-stage breast cancer. Breast Cancer Res Treat. 2016;157(2):339-50.

36. Masuda N, Lee SJ, Ohtani S, Im YH, Lee ES, Yokota I, et al. Adjuvant Capecitabine for Breast Cancer after Preoperative Chemotherapy. N Engl J Med. 2017;376(22):2147-59.

37. von Minckwitz G, Huang CS, Mano MS, Loibl S, Mamounas EP, Untch M, et al. Trastuzumab Emtansine for Residual Invasive HER2-Positive Breast Cancer. N Engl J Med. 2019;380(7):617-28. 
38. Spring LM, Gupta A, Reynolds KL, Gadd MA, Ellisen LW, Isakoff SJ, et al. Neoadjuvant Endocrine Therapy for Estrogen Receptor-Positive Breast Cancer: A Systematic Review and Metaanalysis. JAMA Oncol. 2016;2(11):1477-86.

39. de Glas NA, Kiderlen M, Bastiaannet E, de Craen AJ, van de Water W, van de Velde CJ, et al. Postoperative complications and survival of elderly breast cancer patients: a FOCUS study analysis. Breast Cancer Res Treat. 2013;138(2):561-9.

40. Hind D, Wyld L, Beverley CB, Reed MW. Surgery versus primary endocrine therapy for operable primary breast cancer in elderly women (70 years plus). Cochrane Database Syst Rev. 2006(1):Cd004272.

41. Morgan JL, Reed MW, Wyld L. Primary endocrine therapy as a treatment for older women with operable breast cancer - a comparison of randomised controlled trial and cohort study findings. Eur J Surg Oncol. 2014;40(6):676-84.

42. Syed BM, Al-Khyatt W, Johnston SJ, Wong DW, Winterbottom L, Kennedy H, et al. Longterm clinical outcome of oestrogen receptor-positive operable primary breast cancer in older women: a large series from a single centre. Br J Cancer. 2011;104(9):1393-400.

43. Ward EP, Weiss A, Blair SL. Incidence and treatments of DCIS in octogenarians: grade matters. Breast Cancer Res Treat. 2017;165(2):403-9.

44. Martelli G, Miceli R, Daidone MG, Vetrella G, Cerrotta AM, Piromalli D, et al. Axillary dissection versus no axillary dissection in elderly patients with breast cancer and no palpable axillary nodes: results after 15 years of follow-up. Ann Surg Oncol. 2011;18(1):125-33.

45. Giuliano AE, Ballman KV, McCall L, Beitsch PD, Brennan MB, Kelemen PR, et al. Effect of Axillary Dissection vs No Axillary Dissection on 10-Year Overall Survival Among Women With Invasive Breast Cancer and Sentinel Node Metastasis: The ACOSOG Z0011 (Alliance) Randomized Clinical Trial. Jama. 2017;318(10):918-26.

46. Donker M, van Tienhoven G, Straver ME, Meijnen P, van de Velde CJ, Mansel RE, et al. Radiotherapy or surgery of the axilla after a positive sentinel node in breast cancer (EORTC 1098122023 AMAROS): a randomised, multicentre, open-label, phase 3 non-inferiority trial. Lancet Oncol. 2014;15(12):1303-10.

47. James R, McCulley SJ, Macmillan RD. Oncoplastic and reconstructive breast surgery in the elderly. Br J Surg. 2015;102(5):480-8.

48. McGale P, Taylor C, Correa C, Cutter D, Duane F, Ewertz M, et al. Effect of radiotherapy after mastectomy and axillary surgery on 10-year recurrence and 20-year breast cancer mortality: metaanalysis of individual patient data for 8135 women in 22 randomised trials. Lancet. 2014;383(9935):2127-35.

49. Hughes KS, Schnaper LA, Bellon JR, Cirrincione CT, Berry DA, McCormick B, et al. Lumpectomy plus tamoxifen with or without irradiation in women age 70 years or older with early breast cancer: long-term follow-up of CALGB 9343. J Clin Oncol. 2013;31(19):2382-7.

50. Kunkler IH, Williams LJ, Jack WJ, Cameron DA, Dixon JM. Breast-conserving surgery with or without irradiation in women aged 65 years or older with early breast cancer (PRIME II): a randomised controlled trial. Lancet Oncol. 2015;16(3):266-73.

51. Bartelink H, Maingon P, Poortmans P, Weltens C, Fourquet A, Jager J, et al. Whole-breast irradiation with or without a boost for patients treated with breast-conserving surgery for early breast cancer: 20-year follow-up of a randomised phase 3 trial. Lancet Oncol. 2015;16(1):47-56.

52. Strnad V, Ott OJ, Hildebrandt G, Kauer-Dorner D, Knauerhase H, Major T, et al. 5-year results of accelerated partial breast irradiation using sole interstitial multicatheter brachytherapy versus wholebreast irradiation with boost after breast-conserving surgery for low-risk invasive and in-situ carcinoma of the female breast: a randomised, phase 3, non-inferiority trial. Lancet. 2016;387(10015):229-38.

53. Coles CE, Griffin CL, Kirby AM, Titley J, Agrawal RK, Alhasso A, et al. Partial-breast radiotherapy after breast conservation surgery for patients with early breast cancer (UK IMPORT LOW trial): 5-year results from a multicentre, randomised, controlled, phase 3, non-inferiority trial. Lancet. 2017;390(10099):1048-60.

54. Bloomfield DJ. Development of Postoperative Radiotherapy for Breast Cancer: UK Consensus Statements - a Model of Patient, Clinical and Commissioner Engagement? Clin Oncol (R Coll Radiol). 2017;29(10):639-41. 
55. Poortmans PM, Collette S, Kirkove C, Van Limbergen E, Budach V, Struikmans H, et al. Internal Mammary and Medial Supraclavicular Irradiation in Breast Cancer. N Engl J Med. 2015;373(4):317-27.

56. Whelan TJ, Olivotto IA, Parulekar WR, Ackerman I, Chua BH, Nabid A, et al. Regional Nodal Irradiation in Early-Stage Breast Cancer. N Engl J Med. 2015;373(4):307-16.

57. Bartelink H. Regional nodal irradiation for early breast cancer; clinical benefit according to risk stratification. Breast. 2019;48 Suppl 1:S65-s8.

58. (NICE) NIfHaCE. Early and locally advanced breast cancer: diagnosis and management. 2018. 59. Recht A, Comen EA, Fine RE, Fleming GF, Hardenbergh PH, Ho AY, et al. Postmastectomy Radiotherapy: An American Society of Clinical Oncology, American Society for Radiation Oncology, and Society of Surgical Oncology Focused Guideline Update. Pract Radiat Oncol. 2016;6(6):e219-e34. 60. Kunkler IH, Canney P, van Tienhoven G, Russell NS. Elucidating the role of chest wall irradiation in 'intermediate-risk' breast cancer: the MRC/EORTC SUPREMO trial. Clin Oncol (R Coll Radiol). 2008;20(1):31-4.

61. Gradishar WJ, Anderson BO, Balassanian R, Blair SL, Burstein HJ, Cyr A, et al. NCCN Guidelines Insights: Breast Cancer, Version 1.2017. J Natl Compr Canc Netw. 2017;15(4):433-51.

62. Murray Brunt A, Haviland JS, Wheatley DA, Sydenham MA, Alhasso A, Bloomfield DJ, et al. Hypofractionated breast radiotherapy for 1 week versus 3 weeks (FAST-Forward): 5-year efficacy and late normal tissue effects results from a multicentre, non-inferiority, randomised, phase 3 trial. The Lancet. 2020;395(10237):1613-26.

63. Muss HB, Polley MC, Berry DA, Liu H, Cirrincione CT, Theodoulou M, et al. Randomized Trial of Standard Adjuvant Chemotherapy Regimens Versus Capecitabine in Older Women With Early Breast Cancer: 10-Year Update of the CALGB 49907 Trial. J Clin Oncol. 2019;37(26):2338-48.

64. Elkin EB, Hurria A, Mitra N, Schrag D, Panageas KS. Adjuvant chemotherapy and survival in older women with hormone receptor-negative breast cancer: assessing outcome in a population-based, observational cohort. J Clin Oncol. 2006;24(18):2757-64.

65. Giordano SH, Duan Z, Kuo YF, Hortobagyi GN, Goodwin JS. Use and outcomes of adjuvant chemotherapy in older women with breast cancer. J Clin Oncol. 2006;24(18):2750-6.

66. Tamirisa N, Lin H, Shen Y, Shaitelman SF, Sri Karuturi M, Giordano SH, et al. Association of Chemotherapy With Survival in Elderly Patients With Multiple Comorbidities and Estrogen ReceptorPositive, Node-Positive Breast Cancer. JAMA Oncol. 2020.

67. Kizy S, Altman AM, Marmor S, Denbo JW, Jensen EH, Tuttle TM, et al. 21-gene recurrence score testing in the older population with estrogen receptor-positive breast cancer. J Geriatr Oncol. 2019;10(2):322-9.

68. de Glas NA, van de Water W, Engelhardt EG, Bastiaannet E, de Craen AJ, Kroep JR, et al. Validity of Adjuvant! Online program in older patients with breast cancer: a population-based study. Lancet Oncol. 2014;15(7):722-9.

69. de Glas NA, Bastiaannet E, Engels CC, de Craen AJ, Putter H, van de Velde CJ, et al. Validity of the online PREDICT tool in older patients with breast cancer: a population-based study. Br J Cancer. 2016;114(4):395-400.

70. Muss HB, Woolf S, Berry D, Cirrincione C, Weiss RB, Budman D, et al. Adjuvant chemotherapy in older and younger women with lymph node-positive breast cancer. Jama. 2005;293(9):1073-81.

71. Peto R, Davies C, Godwin J, Gray R, Pan HC, Clarke M, et al. Comparisons between different polychemotherapy regimens for early breast cancer: meta-analyses of long-term outcome among 100,000 women in 123 randomised trials. Lancet. 2012;379(9814):432-44.

72. Perrone F, Nuzzo F, Di Rella F, Gravina A, Iodice G, Labonia V, et al. Weekly docetaxel versus CMF as adjuvant chemotherapy for older women with early breast cancer: final results of the randomized phase III ELDA trial. Ann Oncol. 2015;26(4):675-82.

73. Caparica R, Bruzzone M, Poggio F, Ceppi M, de Azambuja E, Lambertini M. Anthracycline and taxane-based chemotherapy versus docetaxel and cyclophosphamide in the adjuvant treatment of HER2-negative breast cancer patients: a systematic review and meta-analysis of randomized controlled trials. Breast Cancer Res Treat. 2019;174(1):27-37. 
74. Muss HB, Berry DA, Cirrincione C, Budman DR, Henderson IC, Citron ML, et al. Toxicity of Older and Younger Patients Treated With Adjuvant Chemotherapy for Node-Positive Breast Cancer: The Cancer and Leukemia Group B Experience. Journal of Clinical Oncology. 2007;25(24):3699-704. 75. Brouwers B, Hatse S, Dal Lago L, Neven P, Vuylsteke P, Dalmasso B, et al. The impact of adjuvant chemotherapy in older breast cancer patients on clinical and biological aging parameters. Oncotarget. 2016;7(21):29977-88.

76. Cameron D, Piccart-Gebhart MJ, Gelber RD, Procter M, Goldhirsch A, de Azambuja E, et al. 11 years' follow-up of trastuzumab after adjuvant chemotherapy in HER2-positive early breast cancer: final analysis of the HERceptin Adjuvant (HERA) trial. Lancet. 2017;389(10075):1195-205.

77. Perez EA, Romond EH, Suman VJ, Jeong JH, Sledge G, Geyer CE, Jr., et al. Trastuzumab plus adjuvant chemotherapy for human epidermal growth factor receptor 2-positive breast cancer: planned joint analysis of overall survival from NSABP B-31 and NCCTG N9831. J Clin Oncol. 2014;32(33):3744-52.

78. Brain E, Caillet P, de Glas N, Biganzoli L, Cheng K, Lago LD, et al. HER2-targeted treatment for older patients with breast cancer: An expert position paper from the International Society of Geriatric Oncology. J Geriatr Oncol. 2019;10(6):1003-13.

79. Sawaki M, Taira N, Uemura Y, Saito T, Baba S, Kobayashi K, et al. Randomized Controlled Trial of Trastuzumab With or Without Chemotherapy for HER2-Positive Early Breast Cancer in Older Patients. J Clin Oncol. 2020:Jco2000184.

80. Reeder-Hayes KE, Meyer AM, Hinton SP, Meng K, Carey LA, Dusetzina SB. Comparative Toxicity and Effectiveness of Trastuzumab-Based Chemotherapy Regimens in Older Women With Early-Stage Breast Cancer. J Clin Oncol. 2017;35(29):3298-305.

81. Vaz-Luis I, Keating NL, Lin NU, Lii H, Winer EP, Freedman RA. Duration and toxicity of adjuvant trastuzumab in older patients with early-stage breast cancer: a population-based study. J Clin Oncol. 2014;32(9):927-34.

82. Christiansen P, Bjerre K, Ejlertsen B, Jensen MB, Rasmussen BB, Laenkholm AV, et al. Mortality rates among early-stage hormone receptor-positive breast cancer patients: a population-based cohort study in Denmark. Journal of the National Cancer Institute. 2011;103(18):1363-72.

83. Aromatase inhibitors versus tamoxifen in early breast cancer: patient-level meta-analysis of the randomised trials. Lancet. 2015;386(10001):1341-52.

84. Rossi L, McCartney A, De Santo I, Risi E, Moretti E, Malorni L, et al. The optimal duration of adjuvant endocrine therapy in early luminal breast cancer: A concise review. Cancer Treat Rev. 2019;74:29-34.

85. O'Carrigan B, Wong MH, Willson ML, Stockler MR, Pavlakis N, Goodwin A. Bisphosphonates and other bone agents for breast cancer. Cochrane Database Syst Rev. 2017; 10(10):Cd003474.

86. Adjuvant bisphosphonate treatment in early breast cancer: meta-analyses of individual patient data from randomised trials. Lancet. 2015;386(10001):1353-61.

87. Coleman R, Woodward E, Brown J, Cameron D, Bell R, Dodwell D, et al. Safety of zoledronic acid and incidence of osteonecrosis of the jaw (ONJ) during adjuvant therapy in a randomised phase III trial (AZURE: BIG 01-04) for women with stage II/III breast cancer. Breast Cancer Res Treat. 2011;127(2):429-38.

88. Mauri D, Valachis A, Polyzos IP, Polyzos NP, Kamposioras K, Pesce LL. Osteonecrosis of the jaw and use of bisphosphonates in adjuvant breast cancer treatment: a meta-analysis. Breast Cancer Res Treat. 2009;116(3):433-9.

89. Gnant M, Pfeiler G, Steger GG, Egle D, Greil R, Fitzal F, et al. Adjuvant denosumab in postmenopausal patients with hormone receptor-positive breast cancer (ABCSG-18): disease-free survival results from a randomised, double-blind, placebo-controlled, phase 3 trial. Lancet Oncol. 2019;20(3):339-51.

90. Coleman RE, Finkelstein D, Barrios CH, Martin M, Iwata H, Glaspy JA, et al. Adjuvant denosumab in early breast cancer: First results from the international multicenter randomized phase III placebo controlled D-CARE study. Journal of Clinical Oncology. 2018;36(15_suppl):501-.

91. Lichtman SM, Wildiers H, Chatelut E, Steer C, Budman D, Morrison VA, et al. International Society of Geriatric Oncology Chemotherapy Taskforce: evaluation of chemotherapy in older patients-an analysis of the medical literature. J Clin Oncol. 2007;25(14):1832-43. 
92. Biganzoli L, Cinieri S, Berardi R, Pedersini R, McCartney A, Minisini AM, et al. EFFECT: a randomized phase II study of efficacy and impact on function of two doses of nab-paclitaxel as firstline treatment in older women with advanced breast cancer. Breast Cancer Res. 2020;22(1):83.

93. Leo S, Arnoldi E, Repetto L, Coccorullo Z, Cinieri S, Fedele P, et al. Eribulin Mesylate as Third or Subsequent Line Chemotherapy for Elderly Patients with Locally Recurrent or Metastatic Breast Cancer: A Multicentric Observational Study of GIOGer (Italian Group of Geriatric Oncology)ERIBE. Oncologist. 2019;24(6):e232-e40.

94. Wildiers H, Tryfonidis K, Dal Lago L, Vuylsteke P, Curigliano G, Waters S, et al. Pertuzumab and trastuzumab with or without metronomic chemotherapy for older patients with HER2-positive metastatic breast cancer (EORTC 75111-10114): an open-label, randomised, phase 2 trial from the Elderly Task Force/Breast Cancer Group. Lancet Oncol. 2018;19(3):323-36.

95. Rugo HS, Turner NC, Finn RS, Joy AA, Verma S, Harbeck N, et al. Palbociclib plus endocrine therapy in older women with HR+/HER2- advanced breast cancer: a pooled analysis of randomised PALOMA clinical studies. Eur J Cancer. 2018;101:123-33.

96. Sonke GS, Hart LL, Campone M, Erdkamp F, Janni W, Verma S, et al. Ribociclib with letrozole vs letrozole alone in elderly patients with hormone receptor-positive, HER2-negative breast cancer in the randomized MONALEESA-2 trial. Breast Cancer Res Treat. 2018;167(3):659-69.

97. Goetz MP, Okera M, Wildiers H, editors. Safety and Efficacy of Abemaciclib plus endocrine therapy in Elderly patients with HR+, HER2- advanced breast cancer: an age-specific subgroup analysis of MONARCH2 and 3 trials. San Antonio Breast Cancer Symposium; 2019; San Antonio, TX.

98. Howie LJ, Singh H, Bloomquist E, Wedam S, Amiri-Kordestani L, Tang S, et al. Outcomes of Older Women With Hormone Receptor-Positive, Human Epidermal Growth Factor Receptor-Negative Metastatic Breast Cancer Treated With a CDK4/6 Inhibitor and an Aromatase Inhibitor: An FDA Pooled Analysis. J Clin Oncol. 2019;37(36):3475-83.

99. Battisti NML, De Glas N, Sedrak MS, Loh KP, Liposits G, Soto-Perez-de-Celis E, et al. Use of cyclin-dependent kinase 4/6 (CDK4/6) inhibitors in older patients with ER-positive HER2-negative breast cancer: Young International Society of Geriatric Oncology review paper. Ther Adv Med Oncol. 2018;10:1758835918809610.

100. Pritchard KI, Burris HA, 3rd, Ito Y, Rugo HS, Dakhil S, Hortobagyi GN, et al. Safety and efficacy of everolimus with exemestane vs. exemestane alone in elderly patients with HER2-negative, hormone receptor-positive breast cancer in BOLERO-2. Clin Breast Cancer. 2013;13(6):421-32.e8. 